\title{
Phylogenetic analyses of mitochondrial and nuclear data in haematophagous flies support the paraphyly of the genus Stomoxys (Diptera: Muscidae)
}

\author{
Najla Dsouli ${ }^{\mathrm{a}, *}$, Frédéric Delsuc $^{\mathrm{b}}$, Johan Michaux ${ }^{\mathrm{c}}$, Eric De Stordeur ${ }^{\mathrm{a}}$, Arnaud Couloux ${ }^{\mathrm{d}}$, \\ Michel Veuille ${ }^{\mathrm{e}}$, Gérard Duvallet ${ }^{\mathrm{a}}$ \\ ${ }^{a}$ Centre d'Ecologie fonctionnelle et évolutive UMR 5175 CEFE, Université Montpellier 3, Route de Mende, 34199 Montpellier Cedex 5, France \\ ${ }^{\mathrm{b}}$ Institut des Sciences de l'Evolution, UMR 5554-CNRS, Université Montpellier 2, Montpellier, France \\ ${ }^{\mathrm{C}}$ Centre de Biologie et de Gestion des Populations, Montpellier, France \\ d Centre National de Séquençage, Evry Cedex, France \\ e Biologie Intégrative des Populations, Muséum National d'Histoire Naturelle, Paris, France
}

\section{A R T I C L E I N F O}

\section{Article history:}

Received 16 June 2010

Received in revised form 4 February 2011

Accepted 7 February 2011

Available online 13 February 2011

\section{Keywords:}

Stomoxys flies

Phylogenetic relationship

Molecular dating

\begin{abstract}
A B S T R A C T
The genus Stomoxys Geoffroy (Diptera; Muscidae) contains species of parasitic flies that are of medical and economic importance. We conducted a phylogenetic analysis including 10 representative species of the genus including multiple exemplars, together with the closely related genera Prostomoxys Zumpt, Haematobosca Bezzi, and Haematobia Lepeletier \& Serville. Phylogenetic relationships were inferred using maximum likelihood and Bayesian methods from DNA fragments from the cytochrome c oxidase subunit I (COI, 753 bp) and cytochrome b (CytB, 587 bp) mitochondrial genes, and the nuclear ribosomal internal transcribed spacer 2 (ITS2, 426 bp). The combination of mitochondrial and nuclear data strongly supports the paraphyly of the genus Stomoxys because of the inclusion of Prostomoxys saegerae Zumpt. This unexpected result suggests that Prostomoxys should be renamed into Stomoxys. Also, the deep molecular divergence observed between the subspecies Stomoxys niger niger Macquart and $S$. niger bilineatus Grünbreg led us to propose that they should rather be considered as distinct species, in agreement with ecological data. Bayesian phylogenetic analyses support three distinct lineages within the genus Stomoxys with a strong biogeographical component. The first lineage consists solely of the divergent Asian species S. indicus Picard which appears as the sister-group to all remaining Stomoxys species. The second clade groups the strictly African species Stomoxys inornatus Grünbreg, Stomoxys transvittatus Villeneuve, Stomoxys omega Newstead, and Stomoxys pallidus Roubaud. Finally, the third clade includes both African occurring and more widespread species such as the livestock pest Stomoxys calcitrans Linnaeus. Divergence time estimates indicate that the genus Stomoxys originated in the late Oligocene around 30 million years ago, with the major lineages diversifying in the Early Miocene between 20 and 15 million years ago at a time when temperate forests developed in the Northern Hemisphere.
\end{abstract}

(C) 2011 Elsevier B.V. All rights reserved.

\section{Introduction}

Stomoxys flies are principally haematophagous, and are associated with livestock and wildlife throughout the world. The most studied species, Stomoxys calcitrans, is an economically important pest of cattle and several studies have attempted to estimate its impact on cattle production (Miller et al., 1973; Campbell et al., 1977, 2001). These flies represent a serious nuisance not only because of their painful bites and blood predation, but also because they are involved in the mechanical transmission of several pathogens, such as the Capripoxvirus

\footnotetext{
* Corresponding author. Tel.: +330467142315.

E-mail address: dsouli2005@yahoo.fr (N. Dsouli).
}

causing a cattle disease (Lumpy skin disease), Anaplasma marginale the causative agent of severe bovine anaplosmosis, and Dermatophilus congolensis the causative agent of dermotophilosis (Zumpt, 1973; D'Amico et al., 1996; Foil and Gorham, 2000).

The genus Stomoxys belongs to the tribe Stomoxyini in the subfamily Muscinae (De Carvalho, 1989; Couri and De Carvalho, 2003). This subfamily is part of the large family Muscidae comprising about 4500 described species classified in 180 genera (De Carvalho et al., 2005). Within Muscidae, phylogenetic analyses have been conducted at several taxonomic levels to assess the relationships among constitutive species (Couri and Pont, 2000; Couri and De Carvalho, 2003; Schuhli and De Carvalho, 2005; De Carvalho and Pont, 2006; Schuhli et al., 2007). Recently, Nihei and De Carvalho (2007) carried out a cladistic analysis to assess the monophyly of the Muscini tribe. However, since the monograph of 
Table 1

Stomoxys species distributions as mentioned by Zumpt (1973).

\begin{tabular}{|c|c|}
\hline Species & Distribution \\
\hline S. bengalensis Picard & India to Java \\
\hline S. boueti Roubaud & Benin (ex-Dahomey), Congo \\
\hline S. calcitrans (Linnaeus) & Cosmopolitan \\
\hline S. indicus Picard & $\begin{array}{l}\text { Oriental region and neighbouring } \\
\text { Paleartic territories }\end{array}$ \\
\hline S. inornatus Grünberg & Tropical Africa \\
\hline S. luteolus Villeneuve & Central and East Africa \\
\hline \multicolumn{2}{|l|}{ S. niger Macquart } \\
\hline S. niger niger & Ethiopian and Madagascan regions \\
\hline S. niger bilineatus & Ethiopian and Madagascan regions \\
\hline S. ochrosoma Speiser & Central and East Africa \\
\hline S. omega Newstead & Ethiopian Region \\
\hline S. pallidus Roubaud & Tropical Africa \\
\hline S. pullus Austen & India \\
\hline S. sitiens Rondani & $\begin{array}{l}\text { Ethiopian and Oriental regions, } \\
\text { in Egypt it reaches the Palearctic region }\end{array}$ \\
\hline S. stigma Van Emden & Uganda-Congo \\
\hline S. taeniatus Bigot & Ethiopian region \\
\hline S. transvittatus Villeneuve & Southern and Central Africa \\
\hline S. uruma Shinonaga \& Kano & Oriental region \\
\hline S. varipes Bezzi & East and Central Africa southward to Rhodesia \\
\hline S. xanthomelas Roubaud & Congo-Tanzania-Uganda \\
\hline
\end{tabular}

Zumpt (1973) who proposed the monophyly of Stomoxyini on the basis of morphological characters, and despite the medical and economic importance of these parasitic flies, no phylogenetic analysis has been carried out to assess the relationships within Stomoxyini. The flies of this tribe are easily recognized by their typical piercing/sucking mouthparts which differentiate them from the common housefly (Musca domestica) and relatives from the Muscini tribe. The Stomoxyini tribe consists of 10 genera and about 39 species reviewed by Zumpt (1973), the most medically and economically important species are members of the genera Haematobosca, Haematobia and Stomoxys.

The genus Stomoxys, which originated from the old World, includes 18 species (Zumpt, 1973). Among these species, only $S$. calcitrans has a worldwide distribution and is a synanthropic fly. All other species are exclusively tropical, 12 of which are located on the African continent, four on the Asian continent, and one species, Stomoxys sitiens Rondani, has been reported in both Africa and Asia (Zumpt, 1973) (Table 1).

In this study, we address the phylogenetic relationships of the Stomoxys genus using mitochondrial (COI, CytB) and nuclear ribosomal (ITS2) nucleotide sequences. Mitochondrial DNA ( $m$ tDNA) has been widely used in systematic and many universal PCR primers are available for genes like COI and CytB (Avise, 2004). The ITS2 region is easy to amplify and have been used in previous phylogenetic studies of Diptera (Hwang, 2007; Thanwisai et al., 2006). This work explores congruence and information content within the different molecular datasets analysed using probabilistic methods of phylogenetic reconstruction. Phylogenetic results are discussed and taken into consideration to propose a taxonomic revision of the group. Also, we propose estimations of divergence times for major clades within Stomoxyini based on a relaxed molecular clock approach. This allowed discussing the evolutionary history of Stomoxys species in its biogeographical context.

\section{Materials and methods}

\subsection{Taxon sampling}

The material used in this study belongs to the Laboratoire de Zoogéographie of the Université Paul-Valéry in Montpellier (France), and to the Department of Entomology of the Natural History Museum in London (United-Kingdom). For Stomoxys we sampled 11 representative species or subspecies. As outgroups, we incorporated Prostomoxys saegerae, the unique representative species of Prostomoxys, and Haematobosca croceicornis, a newly described species from Gabon (Pont and Dsouli, 2008). For this study, we also included sequences of Haematobia irritans and $M$. domestica (Muscidae), as well as species belonging to the more distantly related Drosophilidae, Syrphidae and Dolichopodidae families in order to provide calibration points for molecular dating analyses (Wiegmann et al., 2003). Geographical origins of studied specimens and sequences accession numbers are listed in Table 2. Specimens are available upon request from the corresponding

Table 2

Species sampling, source localities, and GenBank accession numbers.

\begin{tabular}{|c|c|c|c|c|}
\hline Species & Origin & $\mathrm{COI}$ & CytB & ITS2 \\
\hline \multirow[t]{3}{*}{ Stomoxys calcitrans } & India & EU836073-EU836074 & EU851301 & NA \\
\hline & Thailand & EU836075-EU836082 & EU851303-EU851308 & EU851200-EU851208 \\
\hline & Cameroun & EU836070 & EU851302 & EU851199 \\
\hline Stomoxys indicus & Thailand & EU836083-EU836092 & EU851309-EU851318 & EU851209-EU851218 \\
\hline \multirow[t]{2}{*}{ Stomoxys sitiens } & Thailand & EU836131-EU836138 & EU851357-EU851364 & EU851251-EU851257 \\
\hline & Burkina Faso & FJ386382 & FJ386381 & NA \\
\hline Stomoxys bengalensis & Thailand & EU836060-EU836068 & EU851281-EU851288 & EU851191-EU851198 \\
\hline Stomoxys niger niger & Gabon & EU836110-EU836115 & EU851327-EU851338 & EU851228-EU851237 \\
\hline Stomoxys niger bilineatus & Gabon & EU836101-EU836108 & EU851289-EU851295 & EU851219-EU851227 \\
\hline Stomoxys varipes & Ethiopia & EU836147-EU836148 & EU851372-EU851374 & EU851189-EU851190 \\
\hline Stomoxys transvittatus & Gabon & EU836139-EU836145 & EU851365-EU851370 & EU851258-EU851265 \\
\hline Stomoxys inornatus & Gabon & EU836093-EU836100 & EU851319-EU851326 & EU851179-EU851188 \\
\hline Stomoxys omega & Gabon & EU836116-EU836121 & EU851339-EU851346 & EU851238-EU851244 \\
\hline Stomoxys pallidus & Gabon & EU836122-EU836130 & EU851347-EU851356 & EU851245-EU851250 \\
\hline Prostomoxys saegerae & Gabon & EU836055-EU836059 & EU851275-EU851280 & EU851266-EU851270 \\
\hline Haematobosca croceicornis & Gabon & EU836053-EU836054 & EU851273-EU851274 & EU851271-EU851272 \\
\hline Haematobia irritans & - & AY526195* & DQ029097* & DQ437515* \\
\hline Musca domestica & - & AF104622* & DQ657064* & Z28417* \\
\hline Drosophila yakuba & - & NC_001322* & NC_001322* & Z28416* \\
\hline Drosophila melanogaster & - & NC_001709* & NC_001709* & EU306667* \\
\hline Drosophila virilis & - & DQ471577* & AY646771* & Z28415* \\
\hline Cheilosia naruska & - & DQ417498* & NA & FJ028661* \\
\hline Cheilosia longula & - & FJ158631* & NA & FJ158631* \\
\hline Simosyrphus grandicornis & - & NC_008754* & NC_008754* & NA \\
\hline Dolichopus nubilus & - & AY9558244* & AY958244* & NA \\
\hline Dolichopus excisus & - & AY958245* & AY958245* & NA \\
\hline
\end{tabular}

The sequences marked with an asterisk $\left({ }^{*}\right)$ were obtained from GenBank; NA: no available data. 
Table 3

COI, CytB and ITS2 primers used for amplifications and sequencing.

\begin{tabular}{|c|c|c|}
\hline Primers & Sequences $\left(5^{\prime}->3^{\prime}\right)$ & Reference \\
\hline C1-J-2813 (direct) & CAACATTTATTTTGATTTTTTGG & $\begin{array}{l}\text { Simon et al. } \\
(1994,2006)\end{array}$ \\
\hline TL2-N-3014 (reverse) & TCCATTGCACTAATCTGCCATATTA & \\
\hline CB-J10933 (direct) & GTTTTACCTTGAGGACAAATATC & $\begin{array}{l}\text { Simon et al. } \\
(1994)\end{array}$ \\
\hline CB-N11526 (reverse) & TTCAACTGGTCGAGCTCCAATTCA & \\
\hline ITS2A (direct) & TGTGAACTGCAGGACACAT & $\begin{array}{l}\text { Sharpe et al. } \\
(2000)\end{array}$ \\
\hline ITS2B (reverse) & TATGCTTAAATTCAGGGGGT & \\
\hline
\end{tabular}

author and are stored in the Laboratoire de Zoogéographie of the Université Paul-Valéry in Montpellier (France).

\subsection{DNA extraction, amplification and sequencing}

Genomic DNA was extracted using the DNAeasy tissue Kit (QIAGEN) to a final volume of $180 \mu \mathrm{l}$. Amplifications by PCR, using the specific primer pairs described in Table 3, led to amplicons of different lengths for the COI (753 bp), CytB (587 bp) and ITS2 ( $430 \mathrm{bp})$ regions. All PCR amplifications were performed in a $30 \mu \mathrm{l}$ reaction volume containing, at final concentrations, $200 \mu \mathrm{M}$ dNTPs (diNucleotide Tri Phosphate), $10 \times$ buffer, $25 \mu \mathrm{M}$ of each primers and $0.5 \mu \mathrm{l}$ of Taq polymerase (Eurogentec Red GoldStar ${ }^{\circledR}$ ), and $3 \mu \mathrm{l}$ of purified DNA. Thermal cycling conditions for PCR were as followed: initial denaturation at $94^{\circ} \mathrm{C}$ for $4 \mathrm{~min}, 35$ cycles of denaturation at $94{ }^{\circ} \mathrm{C}$ for $40 \mathrm{~s}$, annealing at $48-50{ }^{\circ} \mathrm{C}$ for COI, 57$58{ }^{\circ} \mathrm{C}$ for CytB, and $60-62{ }^{\circ} \mathrm{C}$ for ITS2, and extension at $72{ }^{\circ} \mathrm{C}$ for $1 \mathrm{~min}$. A final elongation step at $72{ }^{\circ} \mathrm{C}$ for $10 \mathrm{~min}$ completed the DNA amplification process. Ten specimens for each species were sequenced using Sanger sequencing on an ABI 3730 automatic sequencer at the Centre National de Séquençage (Génoscope) in Evry (France).

\subsection{Phylogenetic analyses}

The nucleotide sequences from COI, CytB, and ITS2 were automatically aligned using the multiple alignment program ClustalW 1.4 (Thompson et al., 1994) using default parameters. Multiple sequence alignments were then adjusted by visual inspection, taking the sequences of $S$. calcitrans available in GenBank as a reference for each gene portion. Alignments were cleaned from problematic alignment blocks using Gblocks 0.91 (Castresana, 2000) using the following parameters: minimum number of sequences for a conserved position $=38$; minimum number of sequences for a flanking position $=38$; maximum number of contiguous nonconserved positions $=8$; minimum length of a block = 5; allowed gap positions = with half.

Probabilistic analyses were carried out on each individual datasets (COI, CytB, and ITS2). Maximum likelihood (ML) reconstruction was conducted using PAUP* $4.0 \mathrm{~b} 10$ (Swofford, 2002). The best-fitting models of sequence evolution for different partitions were determined based on the Akaike Information Criterion (AIC) as implemented in jModelTest (Posada, 2008) using PHYML (Guindon and Gascuel, 2003) for calculating likelihood scores. ML heuristic searches were conducted with PAUP* using Tree Bisection Reconnection (TBR) branch-swapping on a Neighbor-Joining ( $\mathrm{NJ}$ ) starting tree using the best-fitting model and associated parameters selected by jModelTest. ML bootstrap proportions were obtained by repeating the same ML heuristic search on 100 pseudo-replicated datasets in order to evaluate the confidence for each node of the tree topology.

Crossed statistical SH tests (Shimodaira and Hasegawa, 1999) of congruence between the three genes were performed in PAUP* by
Table 4

Statistical tests of topological congruence among the three genes using crossed SH tests (Shimodaira and Hasegawa, 1999).

\begin{tabular}{|c|c|c|c|c|}
\hline \multirow[t]{2}{*}{ Topologies } & \multicolumn{4}{|l|}{ Datasets } \\
\hline & $\mathrm{CO} 1$ & CYTB & ITS2 & Concatenation \\
\hline $\mathrm{CO} 1$ & 5207.93 & $42.75^{*}$ & $72.91^{*}$ & $70.32^{\mathrm{ns}}$ \\
\hline CYTB & $145.87^{*}$ & 4174.34 & $144.34^{*}$ & $297.63^{*}$ \\
\hline ITS2 & $162.32^{*}$ & $200.42^{*}$ & 2452.65 & 323.39* \\
\hline Concatenation & $12.89^{\text {ns }}$ & $23.69^{\text {ns }}$ & $3.86^{\text {ns }}$ & 12265.83 \\
\hline
\end{tabular}

Log-likelihood values of ML topologies inferred from each individual gene (CO1, CYTB and ITS2) and from their concatenation were computed using each of the four data matrices and then compared with the corresponding highest log-likelihood value (in bold). The difference in log-likelihood values derived from these crossed comparisons are indicated. An asterisk $\left({ }^{*}\right)$ signifies that the tested topology is significantly worse at the $5 \%$ level than the best ML topology inferred from the corresponding dataset. ns means that this difference is not statistically significant.

testing the best ML topology obtained from each gene against the topology inferred from their concatenation. These tests were run using each individual gene dataset and the concatenated dataset (see Table 4 for details).

Bayesian phylogenetic inference was conducted using MrBayes 3.1.2 (Ronquist and Huelsenbeck, 2003). Three a priori partition schemes were tested for analyzing the concatenation of the three genes (COI, CytB, and ITS2): one single partition, a 3 gene partition, and a 7 partition scheme distinguishing 6 partitions for each codon position of the two coding genes ( $\mathrm{COI}$ and $\mathrm{CytB}$ ) plus a single partition for ITS2. For each partition, we used the best-fitting model selected by jModelTest. Bayesian inference under each partition scheme was conducted with two independent runs of four incrementally heated Metropolis Coupled Markov Chain Monte Carlo (MCMCMC) starting from a random tree. MCMCMC were run for 3,000,000 generations with trees and associated model parameters being sampled every 300 generations. The initial 2000 trees in each run were discarded as burn-in samples and the harmonic mean of the likelihood was calculated by combining the two independent runs. The harmonic means were then used to compute the Bayes factor for the three possible partition comparisons (no partition versus 3 partitions by gene, no partition versus 7 partitions by codon and gene, and 3 partitions by gene versus 7 partitions by codon and gene). The best partition strategy was then determined by the $2 \ln$ Bayes factor criterion as recommended by Brandley et al. (2005). The 50\% majority-rule consensus tree was then computed from the 16,000 trees sampled in the two independent runs under the best model. Posterior probabilities greater than or equal to $95 \%$ are generally regarded as strong support for a clade (Wilcox et al., 2002), but the correspondence with bootstrap support values is not exact (Douady et al., 2003).

\subsection{Molecular datings}

Bayesian estimation of divergence times under relaxed-clock models was conducted using the Multidivtime package (Thorne and Kishino, 2002). We used the previously estimated Bayesian topology as the best hypothesis for Stomoxys phylogeny onto which divergence dates were estimated. The inclusion of species belonging to Drosophilidae (Drosophila virilis, Drosophila melanogaster and Drosophila yakuba), Syrphidae (Simosyrphus grandicornis, Cheilosia longula and Cheilosia naruska) and Dolichopodidae (Dolichopus longula and Dolichopus nubilus), which represent other brachyceran groups, allowed calibrating the tree. Indeed, based on a previously established timescale for brachyceran flies from Bayesian analyses of 28S rRNA data (Wiegmann et al., 2003), our tree was calibrated by using (1) the occurrence of Schizophora 87 million years ago (Mya) as the a priori expected number of time 
units between tip and root, and (2) the estimated date of the Drosophila/Musca split between 48 and 51 Mya as the calibration constraint. This previous molecular estimate was used in the absence of any relevant fossil calibration for Stomoxyini.

First, the program Baseml of the PAML package version $4.2 \mathrm{~b}$ (Yang, 2007) was used to estimate the nucleotide frequencies, transition/transversion ratio, and rate heterogeneity among sites for the concatenated dataset. These values parameterize the F84 + G nucleotide substitution model. Second, we calculated the branch lengths of the constrained topology and the associated variance-covariance matrix under this model using with the program Estbranches. Finally, the program Multidivtime was used to run a Markov chain Monte Carlo (MCMC) for estimating mean posterior divergence times on nodes with associated standard deviations and 95\% credibility intervals (95\% CredI) from the variance-covariance matrix produced by Estbranches. The MCMC was sampled 10,000 times every 100 cycles after a burn-in stage of 100,000 cycles. The prior for the expected number of time units between tip and root was set at 87 Mya ( $S D=43$ Mya). The estimated branch lengths obtained by Estbranches were used to estimate the median amount of evolution between the root and all the tips of the ingroup. Other priors for gamma distribution of the rate at root node (rtrate and rtratesd) and the Brownian motion constant describing the rate variation (brownmean and brownsd) were derived from the median branch length of the phylogram. The highest possible number of time units between tip and root (Bigtime) was set to 119 Mya which corresponds to the upper bound of the $95 \%$ CredI for Schizophora as estimated by Wiegmann et al. (2003). The age of the node Musca/Drosophila was constrained using an upper bound of 51 Mya and a lower bound of 48 Mya (Wiegmann et al., 2003).

\section{Results}

\subsection{Sequence characteristics}

Alignment statistics and models selected for phylogenetic analyses for each data partitions are summarized in Table 5 . Alignment of the mitochondrial genes was straightforward, as no indels were introduced. The alignment of $\mathrm{COI}$ sequences resulted in 753 nucleotide sites of which 228 (30.3\%) are variable, and 198 (26.3\%) are parsimony informative. jModelTest identified the GTR + G + I model as the best-fitting model for the COI gene ( $\ln L=-3610.24$ ) based on the AIC. The gamma distribution shape parameter (alpha) was estimated to 0.2 which reveals strong among-site rate heterogeneity in this barcoding gene. The CytB alignment contained 587 sites of which 204 (34.8\%) are variable and $161(27.4 \%)$ are informative. jModelTest also identified the GTR + G + I model as the best-fitting nucleotide substitution model for the CytB gene $(\ln L=-3121.46)$ with an estimated alpha of 0.62 . Finally, the initial alignment of ITS2 sequences totalized 426

Table 5

Best fitting models and associated maximum likelihood parameters obtained for the gene partitions used in this study.

\begin{tabular}{lrlllllll}
\hline Dataset & Sites & A & T & C & G & Best model & Alpha & Pinv \\
\hline COI & 753 & 0.31 & 0.41 & 0.14 & 0.14 & GTR + I+G & 0.2 & 0.01 \\
First pos. & 251 & 0.46 & 0.49 & 0.04 & 0.01 & TrN + G & 0.45 & - \\
Second pos. & 251 & 0.29 & 0.32 & 0.12 & 0.27 & GTR + G & 0.16 & - \\
Third pos. & 251 & 0.21 & 0.41 & 0.24 & 0.14 & HKY + I & - & 0.80 \\
CytB & 587 & 0.32 & 0.42 & 0.14 & 0.12 & GTR + I+G & 0.62 & 0.35 \\
First pos. & 196 & 0.30 & 0.32 & 0.16 & 0.21 & TIM + G & 0.24 & - \\
Second pos. & 196 & 0.20 & 0.46 & 0.29 & 0.11 & HKY + I & - & 0.68 \\
Third pos. & 195 & 0.45 & 0.48 & 0.06 & 0.01 & HKY + G & 0.77 & - \\
ITS2 & 295 & 0.36 & 0.37 & 0.10 & 0.17 & HKY + G & 0.43 & - \\
Concatenation & 1635 & 0.33 & 0.11 & 0.12 & 0.44 & GTR + I + G & 0.95 & 0.44 \\
\hline
\end{tabular}

sites including indels. 131 sites corresponding to ambiguously aligned hypervariable regions were excluded from subsequent analyses by applying Gblocks. The remaining 295 sites contain 168 (57\%) variable sites of which 147 (50\%) are parsimony informative which makes this marker the most variable of our study. jModelTest identified the HKY + G model as the best-fitting model for the ITS2 marker ( $\ln L=-2454.70)$. The gamma distribution shape parameter alpha was estimated to 0.43 in this ribosomal internal transcribed spacer.

\subsection{Phylogenetic relationships}

We evaluated topological congruence among the individual genes by computing crossed $\mathrm{SH}$ tests in which the highestlikelihood topologies obtained with each individual datasets and their concatenation were compared against each other (Table 4). While there is apparent incongruence among the individual markers, none of the three individual datasets in fact significantly rejects the ML topology supported by their concatenation (see last line of Table 4). This indicates that combining the three individual gene datasets leads to a phylogenetic estimate that is compatible with the signal contributed by each individual gene. We therefore decided to concatenate the three datasets in order to maximize the number of characters analysed and to enhance the phylogenetic signal as advocated by Delsuc et al. (2002).

The total number of nucleotide positions in the concatenation was 1635. Bayes factor comparisons showed that the best-fitting model for the whole concatenation was the use of a single GTR + G model. Indeed, the $2 \ln$ Bayes factor criterion (Brandley et al., 2005) was always in favour of $\mathrm{HO}$ in the three partition scheme comparisons $(2 \ln B F<0.03)$ indicating that it was not worth partitioning in our case. The phylogenies obtained from ML and Bayesian methods under a single GTR $+G$ were identical, but the Bayesian analysis provided higher support values for most of the nodes (Fig. 1). Our results support the monophyly of Haematobia and Haematobosca ( $\left.\mathrm{PP}=1.0 ; \mathrm{BP}_{\mathrm{ML}}=71\right)$, and this clade appears as the sister-group to the remaining Stomoxyini. The genus Stomoxys is rendered paraphyletic with strong support $\left(\mathrm{PP}=1.0 ; \mathrm{BP}_{\mathrm{ML}}=90\right)$ due to the inclusion of $P$. saegerae within it, as a sister-group to Stomoxys varipes (Fig. 1). Strong support is obtained ( $P P=1.00$; $\mathrm{BP}_{\mathrm{ML}}=97$ ) for $S$. indicus as representing the sister-group to all remaining species within the Stomoxys group (Fig. 1). Three major clades can be distinguished according to the Bayesian consensus tree within Stomoxyini. The first clade (clade A) is represented by $S$. indicus alone which is an Asian species. The second clade (clade B) is well supported ( $\mathrm{PP}=0.99$ and $\mathrm{BP}_{\mathrm{ML}}=63$ ) and groups species from African origin (Stomoxys pallidus, Stomoxys omega, Stomoxys transvittatus, and Stomoxys inornatus). Finally, the third clade (clade C), only supported by the Bayesian analysis ( $P P=0.92)$, regroups all the remaining species containing cosmopolitan, African, and Asian species. This clade can be further divided into three subclades. The first subclade associates the two subspecies Stomoxys niger niger and $S$. niger bilineatus $(\mathrm{PP}=0.98)$, the second subclade associates $P$. saegerae and $S$. varipes $(P P=0.89)$, and the third subclade composed of S. calcitrans, S. sitiens, and Stomoxys bengalensis $\left(\mathrm{PP}=1.0 ; \mathrm{BP}_{\mathrm{ML}}=56\right)$ with $S$. sitiens and $S$. bengalensis being sister-groups $\left(\mathrm{PP}=1.0 ; \mathrm{BP}_{\mathrm{ML}}=91\right)($ Fig. 1$)$.

\subsection{Estimation of divergence times}

The divergence time estimates are presented as a chronogram where branching nodes correspond to the mean age estimated from the posterior distribution and its associated 95\% CredI (Fig. 2). The divergence time between the Stomoxys genus and its Haematobia + Haematobosca sister-clade is estimated around 30.8 Mya (95\% CredI: 40.3-22.3). The age estimate for the early 


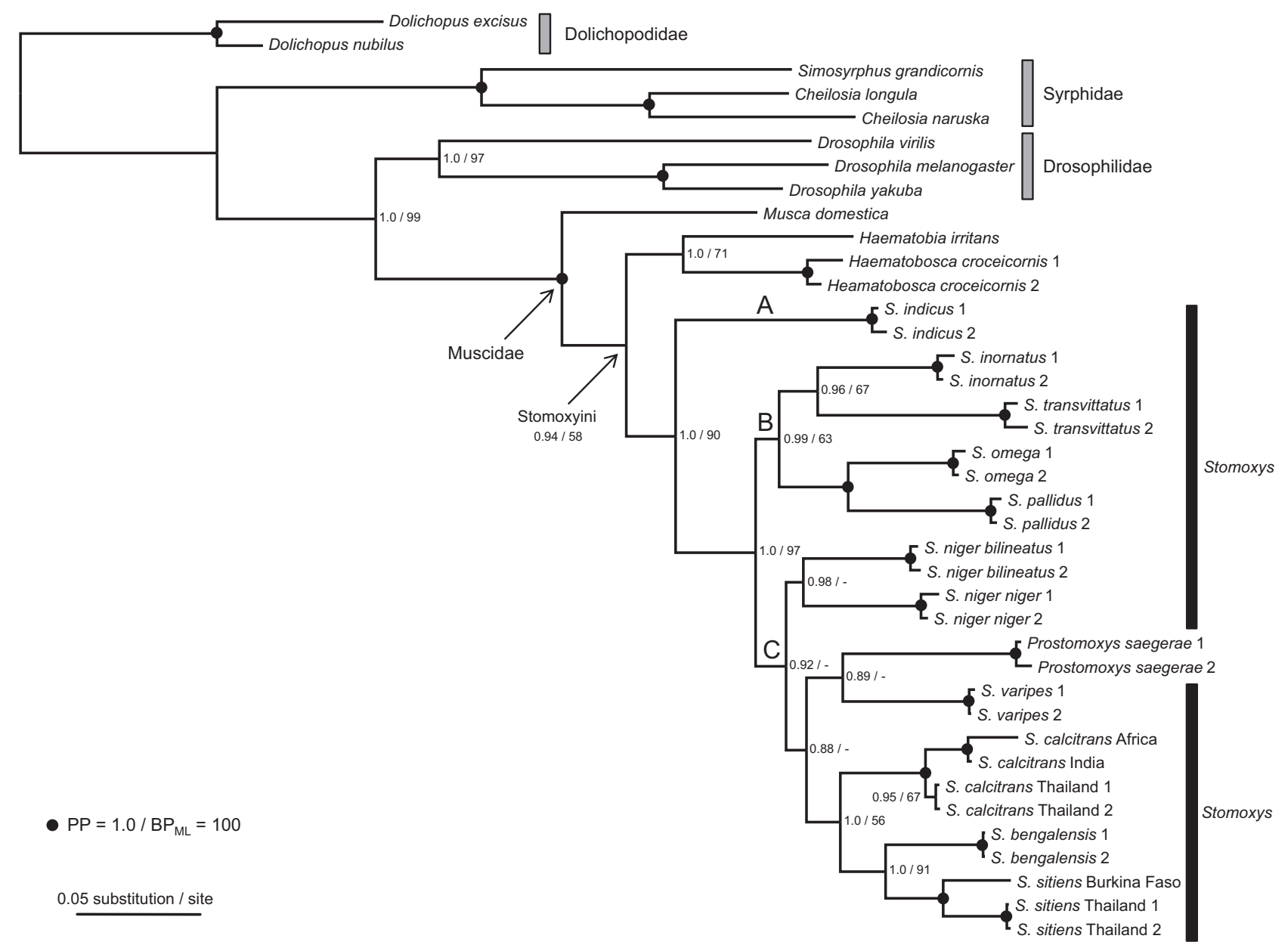

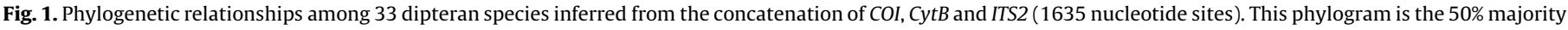

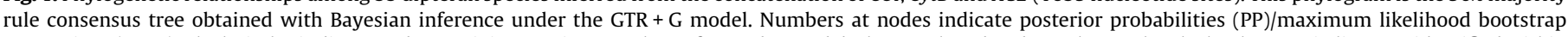

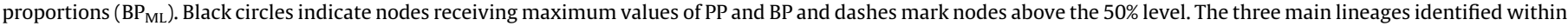
the Stomoxys group are labeled A, B, and C.

emergence of S. indicus (clade A) within the genus Stomoxys is estimated at about 27 Mya (36.6-18.9). The divergence between clade B and clade C occurred around 20.8 Mya (29.6-13.6). According to this inferred timescale, the major lineages within the genus Stomoxys were present by the late Oligocene, while the greatest amount of cladogenesis occurred during the Early Miocene. The two subspecies of $S$. niger ( $S$. niger niger and S. niger bilineatus) separated around 16.3 Mya (24.2-10.3), a divergence time almost exactly similar to the one inferred for the separation between the species $S$. inornatus and $S$. transvittatus estimated at 16.4 Mya (24.6-10.0). Finally, the divergence between $P$. saegerae and $S$. varipes is estimated to have occurred 14.2 Mya (21.6-8.5) concomitantly with the separation of $S$. calcitrans from its $S$. bengalensis/S. sitiens sister-clade at 14.1 Mya (21.5-8.5).

\section{Discussion}

\subsection{Phylogenetic relationships and taxonomy of the Stomoxys group}

The phylogenetic analyses performed in the present study allowed reconstructing a phylogenetic framework for the major constitutive species of the genus Stomoxys and closely related genera. Our analyses strongly support the monophyly of Stomoxyini including a monophyletic group consisting of Haematobia and Haematobosca as a sister-clade to all remaining Stomoxyini. However, according to our results the genus Stomoxys sensu stricto is found to be paraphyletic due to the unexpected position of $P$. saegerae which appears to be well nested within the Stomoxys group as a sister-group to $S$. varipes (Fig. 1). Zumpt (1973) created the genus Prostomoxys for the sole species P. saegerae. According to Zumpt's identification key for Stomoxyini, Prostomoxys is characterized by maxillary palps that are as long as the proboscis, whereas Stomoxys is characterized by palps that are shorter than half the length of the proboscis. This key includes no other diagnostic character for the distinction of this genus from Stomoxys. Zumpt (1973) mentioned that the plesiomorphic form of the Stomoxyini mouthparts can be accepted as the maxillary palps being about as long as the proboscis. Based on our phylogenetic study there appears to be no reason to incorporate this species in a separate genus as $P$. saegerae is in fact closely related to Stomoxys species. Prostomoxys should thus be synonymised with Stomoxys, and the species $P$. saegerae must be renamed into Stomoxys saegerae. The long palp characters would be better considered as mere specific traits, since they are likely to be plesiomorphic.

Within Stomoxys lato sensu (including $P$. saegerae), our phylogenetic analyses strongly support the placement of $S$. indicus as the sister-group to the remaining Stomoxys species. Taxonomically, S. indicus is considered to be highly variable, with a number of synonyms used in the entomological literature. As mentioned by Zumpt (1973): "it is possible that a numerical taxonomic study based on great numbers of specimens from various populations 


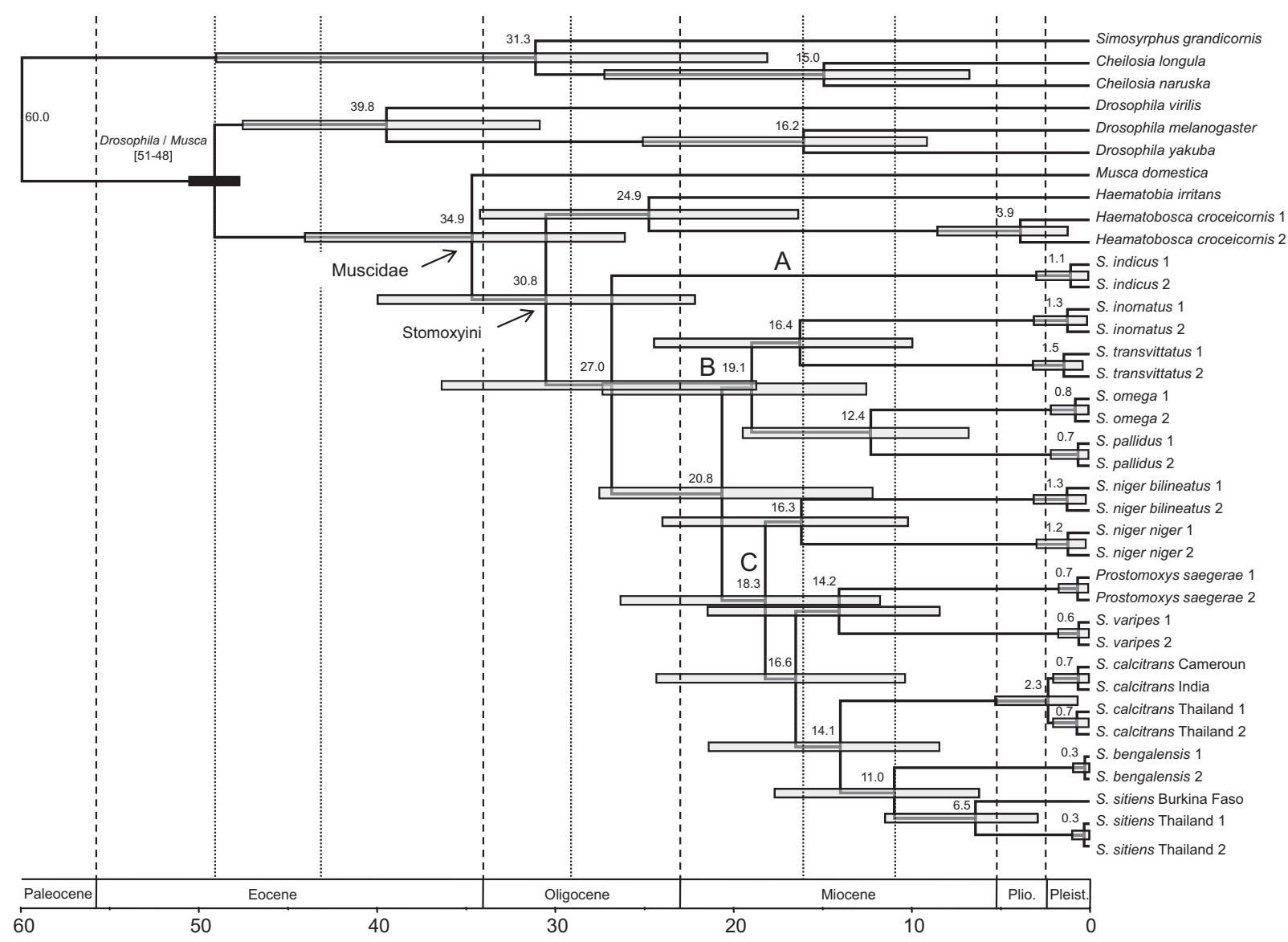

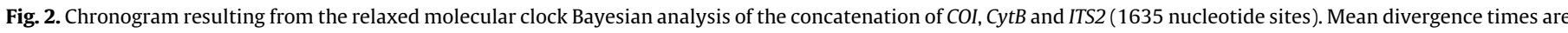

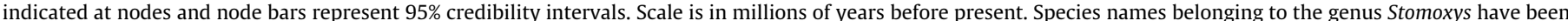

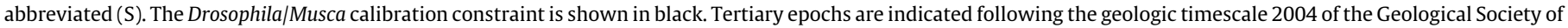
America (Gradstein et al., 2004). Plio.: pliocene; Pleist.: pleistocene.

may reveal that several subspecies may be retained in the future". This species is described as the most common Stomoxys species in the Oriental region after $S$. calcitrans and has been recorded from India to the Pacific islands of Fiji and Samoa, including China and Japan. Its geographic range also reaches the Palaearctic region on the occidental side. Moreover, it has also been recorded in a number of South-East Asian countries such as Thailand (Masmeatathip et al., 2006), Malaysia, Viet Nam, Taiwan, Borneo, Sumatra, and Java (Zumpt, 1973). Since both of our samples are from Thailand origin, they provide no information on the genetic diversity among populations of the species, but they reveal its evolutionary distinctiveness from other members of the genus Stomoxys.

The remaining Stomoxys species included in our study can be divided in two main clades. Clade B contains only African species and clade $\mathrm{C}$ groups both African and more geographically widespread species (Fig. 1). Clade B regroups S. inornatus, S. transvitattus, S. omega and S. pallidus sampled from different parts of the Ethiopian region. These species are ubiquitous and are restricted mainly to forest ecotones where they feed especially on wildlife fauna (Mavoungou et al., 2008). This large clade appears to be divided in two main subclades associating $S$. inornatus and $S$. transvittatus on one side, and S. omega and S. pallidus, on the other side. S. inornatus and S. transvittatus have a diurnal activity whereas S. omega and S. pallidus have a crepuscular activity (Duvallet, unpublished data). These observations would fit well with the proposed phylogeny. However, the information content of ecological data for phylogeny is probably limited since activity levels in Stomoxys have been shown to be highly dependent upon geographical origin (primary forest, secondary forest and humanmodified area) (Mavoungou et al., 2008), climate (temperature, humidity, and solar radiation level) (Kangwagye, 1974; Charlwood and Lopes, 1980), trapping method, and physiological state of individuals (Simmons, 1944; La Breque et al., 1975).

The third clade $C$ can be divided into three subclades, two of them being exclusively African, and one being more geographically widespread. The first subclade groups $S$. niger niger and $S$. niger bilineatus, considered as two subspecies of $S$. niger according to Zumpt (1973), whereas the second subclade associates $P$. saegerae and $S$. varipes. Solving species boundaries between closely related species is notoriously difficult in these parasitic flies. Based on the tree topology, S. niger niger and S. niger bilineatus sequences form distinct phylogenetic clusters. The pairwise distance between $S$. niger niger and S. niger bilineatus based on COI sequences is $8.2 \%$. The use of DNA sequences for species delimitation has been widely criticized (Tautz et al., 2002; DeSalle et al., 2005; Meier et al., 2006). The wide overlap between intraspecific and interspecific variability observed in Diptera COI sequences (0-15.5\%) (Meier et al., 2006) is especially problematic here, since the pairwise distance for these subspecies falls into this overlapping area. However, the genetic distance between $S$. niger niger and S. niger bilineatus based on the nuclear ITS2 is also quite large with $14.3 \%$. Moreover, our molecular dating analysis estimates the separation between the two subspecies around 16.3 Mya (Fig. 2). This is almost as old as the separation between S. inornatus and S. transvittatus (c.a. 16.4 Mya) and is comparable with the divergence observed between $D$. 
melanogaster and D. yakuba (c.a. 16.2 Mya). It is also more ancient than the divergence between S. omega and S. pallidus (c.a. 12.4 Mya), and even more ancient than the split between $S$. varipes and P. saegerae (c.a. 14.2 Mya) (Fig. 2).

Morphologically, S. niger niger and S. niger bilineatus are distinguished only by the colour of their tibiae and tarsi. Mavoungou et al. (2008) described S. niger bilineatus and S. niger niger as sympatric species, the former being abundant in savannas with abundant wild fauna, while the latter is more associated with anthropized area. Mihok et al. (1996) also pointed out differences in habitat affinities, sex ratio and activity patterns between these species. Given morphological observations, ecological isolation and molecular data, it appears very likely that $S$. niger niger and $S$. niger bilineatus belong to different species. Consequently, we suggest raising them to full specific status and to use $S$. niger and $S$. bilineatus as species names.

Finally, the third subclade within clade $C$ associates $S$. calcitrans, S. sitiens and S. bengalensis Picard. S. bengalensis occurs only in Asia, S. sitiens is found in both the African and Asian continents, while the livestock pest $S$. calcitrans has a worldwide distribution. $S$. calcitrans and $S$. sitiens appeared to be strictly human commensals since they have invariably been found in association with human activity, including inside buildings.

\subsection{Molecular timescale and biogeography of the Stomoxys group}

Our molecular estimate of the separation between Stomoxyini and Muscini falls into the Late Eocene epoch (c.a. 34.9 Mya) (Fig. 2). This divergence time is compatible with the record of the oldest Muscidae fossils in the Eocene and the Lower Miocene, 50-20 Mya (Evenhuis, 1994). The oldest Muscini fossil (about 20-15 Mya) was described from Dominican amber (Pont and De Carvalho, 1997), whereas no Stomoxyini fossil has been reported yet. The results presented here show that the radiation of the major Stomoxyini groups largely overlaps with the hypothetical time span (midOligocene to mid-Miocene) during which temperate forests developed in the Northern Hemisphere (Guo et al., 2002).

The genus Stomoxys appears to diverge from other Stomoxyini genera in the Oligocene (c.a. 30 Mya). An Oriental origin of the Stomoxys genus lato sensu is tentatively suggested, owing to the strong support obtained for the basal branching of S. indicus (clade A) and its absence from Africa. Under such a scenario, the common ancestor of the remaining Stomoxys members would have then split into two groups in the Early Miocene (c.a. 21 Mya), with one group being distributed in the Ethiopian region (clade $B$ ), while the other colonized Asia (clade C). The collision between Eurasia and the Arabo-African plate was initiated by the Oligocene, but the Early Miocene period seems to have been key for the establishment of intercontinental pathways permitting faunal exchanges between the Afro-Arabian and Asia plates (Bernor et al., 1987). This biogeographical event fits well with our estimates of the split between clades B and C occurring within this period. Continental exchanges through migration events continued until the Late Miocene and could explained the occurrence of $S$. calcitrans and $S$. sitiens in both the African and Asian continents. Migration until the Late Miocene may have been facilitated by a forest connection that existed between the African and Oriental regions at this epoch (Moreau, 1963).

Our study is a first step into the molecular phylogenetic analysis of the Stomoxys genus. Yet, our results point to the need for a taxonomic revision of the Stomoxyini tribe. Since very few data on the biology and ecology of this group are currently available it would be necessary to advance the knowledge in these basic research areas. This is a prerequisite for better understanding how these species originated and diversified in a biogeographical context.

\section{Acknowledgements}

We thank Jacques-François Mavoungou (Gabon), Roundthip Masmeatathip (Thailand), Alekaw Sinshaw Tegegne (Ethiopia) and the British Museum for providing some Stomoxys samples. We extend our thanks to Gwenaëlle Mondor (CBGP) for technical assistance. We also thank two anonymous reviewers for constructive comments. This study was partially supported by Agence Nationale de la Recherche through the ANR-Biodiv grant IFORA (Iles Forestières Africaines) and by the CNS-Genoscope "Barcoding insects for identification" sequencing grant to Michel Veuille. During the course of this research, N.D.-A. was partially supported by a grant from Société Entomologique de France. This is contribution ISEM 2011-014 of the Institut des Sciences de l'Evolution (UMR 5554-CNRS).

\section{References}

Avise, J.C., 2004. Molecular Markers, Natural History and Evolution. Chapman \& Hall, New York.

Bernor, R.L., Brunet, M. Ginsburg L, Mein, P. Pickford, M., Rögl, R, Sen, S. Steininger, F., Thomas, H., 1987. A consideration of some major topics concerning Old World Miocene mammalian chronology, migrations and paleogeography. Geobios 20, 431-439.

Brandley, M.C., Schmitz, A., Reeder, T.W., 2005. Partitioned Bayesian analyses, partition choice, and the phylogenetic relationships of scincid lizards. Systematic Biology 54, 373-390.

Campbell, J.B., White, R.G, Wright, J.E., Crookshank, R., Clanton, D.C., 1977. Effects of stable flies (Diptera-Muscidae) on weight gains and feed-efficiency of calves on growing and finishing rations. Journal of Economic Entomology 70, 592-594.

Campbell, J.B., Skoda, S.R., Berkerile, D.R., Boxler, D.J., Thomas, G.D., Adams, D.C. Davis, R., 2001. Effects of stable flies (Diptera: Muscidae) on weight gains of grazing yearling cattle. Journal of Economic Entomology 94, 780-783.

Charlwood, J.D., Lopes, J., 1980. The age-structure and biting behaviour of Stomoxys calcitrans (L.) (Diptera: Muscidae) from Manaus, Brazil. Bulletin of Entomological Research 70, 549-555.

Castresana, J., 2000. Selection of conserved blocks from multiple alignments for their use in phylogenetic analysis. Molecular Biology and Evolution 17, 540552.

Couri, M.S., De Carvalho, C.J.B., 2003. Systematic relations among Philornis Meinert, Passeromyia Rodhain \& Villeneuve and allied genera (Diptera, Muscidae). Brazilian Journal of Biology 63, 223-232.

Couri, M.S., Pont, A.C., 2000. Cladistic analysis of Coenosiini (Diptera: Muscidae: Coenosiinae). Systematic Entomology 25, 373-392.

D'Amico, F., Gouteux, J.P., Le Gall, F., Cuisance, D., 1996. Are stable fly (Diptera: Stomoxyinae) vectors of Trypanosoma vivax in the Central African Republic. Veterinary Research 27, 161-170.

De Carvalho, C.J.B., 1989. Classificação de Muscidae (Diptera): una proposta através da análise cladística. Revista Brasileira de Zoologia 6, 627-648.

De Carvalho, C.J.B., Couri, M.S., Pont, A.C., Pamplona, D.M., Lopes, S.M., 2005. A catalogue of the Muscidae (Diptera) of the Neotropical region. Zootaxa 860, 1282 .

De Carvalho, C.J.B., Pont, A.C., 2006. Taxonomy, cladistics and biogeography of the South American Brachygasterina Macquart (Diptera: Muscidae). Zootaxa 1151, $1-26$.

Delsuc, F. Scally, M., Madsen, O., Stanhope, M.J., de Jong W.W., Catzeflis, F.M. Springer, M.S., Douzery, E.J.P., 2002. Molecular phylogeny of living xenarthrans and the impact of character and taxon sampling on the placental tree rooting. Molecular Biology and Evolution 19, 1656-1671.

DeSalle, R., Egan, M.G., Siddal, M., 2005. The unholy trinity: taxonomy, species delimitation and DNA barcoding. Philosophical Transactions of the Royal Society B: Biological Sciences 360, 1905-1916.

Douady, C.J., Delsuc, F., Boucher, Y., Doolittle, W.F., Douzery, E.J.P. 2003. Comparison of Bayesian and maximum likelihood bootstrap measures of phylogenetic reliability. Molecular Biology and Evolution 20, 248-254.

Evenhuis, N.L., 1994. Catalogue of the Fossil Flies of the World (Insecta: Diptera). Backhuys, Leiden.

Foil, L.D., Gorham, J.R., 2000. Mechanical transmission of disease agents by arthropods. In: Eldridge, B.F., Edman, J.D. (Eds.), Medical Entomology. Kluwer Academic Publishers, Dordrecht, The Netherlands, pp. 461-514.

Gradstein, F.M., Ogg, J.G., Smith, A.G., 2004. A Geologic Timescale 2004. Cambridge University Press, New York.

Guindon, S., Gascuel, O., 2003. A simple, fast and accurate algorithm to estimate large phylogenies by maximum likelihood. Systematic Biology 52, 696-704.

Guo, Z.T., Ruddiman, W.F., Hao, Q.Z., Wu, H.B., Qiao, Y.S., Zhu, R.X., Peng, S.Z., Wei, J.J., Yuan, B.Y., Liu, T.S., 2002. Onset of Asian desertification by 22 Myr ago inferred from loess deposits in China. Nature 416, 159-163.

Hwang, U.W., 2007. Revisited ITS2 phylogeny of Anopheles (Anopheles) Hyrcanus group mosquitoes: re-examination of unidentified and misidentified ITS sequences. Parasitology Research 101 (41), 885-894. 
Kangwagye, T.N., 1974. The seasonal incidence of biting flies (Diptera) in Rwenzori National Park and Kigezi Game Reserve, Uganda. Bulletin of Entomological Research 63, 535-549.

La Breque, G.C., Weidhaas, D.E., Whitfield, T.L., 1975. Graphic models as intermediate steps to computerized simulations of stable fly populations. Mosquito News 35, 316-321.

Masmeatathip, R., Gilles, J., Ketavan, C., Duvallet, G., 2006. First survey of seasonal abundance and daily activity of Stomoxys spp. (Diptera: Muscidae) in Kamphaengsaen campus, Nakornpathom Province Thailand. Parasite 13, 245-250.

Mavoungou, J.F., Jay-Robert, P., Gilles, J., Atsame, E.A., Duvallet, G., 2008. Ecology of Stomoxys flies (Diptera: Muscidae) in Gabon. First survey in different ecological areas. Parasite 15, 27-34.

Meier, R., Shiyang, K., Vaidya, G., Ng, P.K.L., 2006. DNA Barcoding and taxonomy in Diptera: a tale of high intraspecific variability and low identification success. Systematic Biology 55 (5), 715-728.

Mihok, S., Opiyo, M., Eli, M., Khalfan, S., 1996. Phenology of Stomoxinae in a Kenya forest. Medical and Veterinary Entomology 10, 305-316.

Miller, R.W., Pickens, L.G., Morgan, N.O., Thimijan, R.W., Wilson, R.L., 1973. Effect of stable flies on feed intake and milk production of dairy cows. Journal of Economic Entomology 66, 711-713.

Moreau, R.E., 1963. Vicissitudes of the African biomes in the late Pliocene. Proceedings of the Zoological Society of London 141, 395

Nihei, S.S., De Carvalho, C.J.B., 2007. Phylogeny and classification of Muscini (Diptera, Muscidae). Zoological Journal of the Linnean Society 149, 493-532.

Pont, A.C., De Carvalho, C.J.B., 1997. Three species of Muscidae (Diptera) from Dominican amber. Studia Dipterologica 4, 173-181.

Pont, A.C., Dsouli, N., 2008. A new species of Haematobosca Bezzi from Gabon (Diptera: Muscidae). Studia Dipterologica 15 (1/2), 259-266.

Posada, D., 2008. jModelTest: phylogenetic model averaging. Molecular Biology and Evolution 25, 1253-1256.

Ronquist, F., Huelsenbeck, J.P., 2003. MrBayes 3: Bayesian phylogenetic inference under mixed models. Bioinformatics 19, 1572-1574.

Schuhli, G.S., De Carvalho, C.J.B., 2005. Revision and cladistics of the Neotropical genus Pseudoptilolepis Snyder (Diptera, Muscidae). Revista Brasileira de Zoologia 22, 23-34.

Schuhli, G.S., De Carvalho, C.J.B., Wiegmann, B.M., 2007. Molecular phylogenetics of the Muscidae (Diptera: Calyptratae): new ideas in a congruence context Invertebrate Systematics 21, 263-278.

Sharpe, R.G., Harbach, R.E., Butlin, R.K., 2000. Molecular variation and phylogeny of members of the Minimus group of Anopheles subgenus Cellia (Diptera: Culicidae). Systematic Entomology 25, 263-272.
Shimodaira, H., Hasegawa, M., 1999. Multiple comparisons of log-likelihoods with applications to phylogenetic inference. Molecular Biology and Evolution 16, 1114-1116.

Simmons, S.W., 1944. Observations on the biology of the stable fly in Florida. Journal of Economic Entomology 37, 680-686.

Simon, C., Frati, F., Beckenbach, A., Crespi, B., Lui, H., Flook, P., 1994. Evolution, weighting and phylogenetic unity of mitochondrial gene sequences and a compilation of conserved polymerase chain reaction primers. Annals of Entomological Society of America 87, 651-701.

Simon, C., Buckley, T.R., Frati, F., Stewart, J.B., Beckenbach, A.T., 2006. Incorporating molecular evolution into phylogenetic analysis, and a new compilation of conserved polymerase chain reaction primers for animal mitochondrial DNA Supplemental material. Annals of Entomological Society of America 37, 545579.

Swofford, D.L., 2002. PAUP*: Phylogenetic Analysis Using Parsimony (* and Other Methods). Sinauer Associates, Sunderland MA.

Tautz, D., Arctander, P., Minelli, A., Thomas, R.H., Vogler, A.P., 2002. DNA points the way ahead in taxonomy. Nature $418,479$.

Thanwisai, A., Kuvangkadilok, C., Baimai, V., 2006. Molecular phylogeny of black flies (Diptera: Simuliidae) from Thailand, using ITS2rDNA. Genetica 128 (1-3) $177-204$.

Thompson, J.D., Higgins, D.G., Gibson, T.J., 1994. CLUSTAL W: improving the sensitivity of progressive multiple sequence alignment through sequence weighting, position specific gap penalties and weight matrix choice. Nucleic Acids Research 22, 4673-4680.

Thorne, J.L., Kishino, H., 2002. Divergence time and evolutionary rate estimation with multilocus data. Systematic Biology 51, 689-702.

Wiegmann, B.M., Yeates, D.K., Thorne, J.L., Kishino, H., 2003. Time flies, a new molecular time-scale for brachyceran fly evolution without a clock. Systematic Biology 52, 745-756.

Wilcox, T.P.D., Zwickl, J., Heath, T., Hillis, D.M., 2002. Phylogenetic relationships of the dwarf boas and a comparison of Bayesian and bootstrap measures of phylogenetic support. Molecular Phylogenetics and Evolution 25, 361371.

Yang, Z., 2007. PAML 4: a program package for phylogenetic analysis by maximum likelihood. Molecular Biology and Evolution 24, 1586-1591., http://abacus. gene.ucl.ac.uk/software/paml.html.

Zumpt, F., 1973. The Stomoxyinae biting flies of the world. In: Taxonomy, Biology, Economic Importance and Control Measures, Gustav Fischer Verlag, Stuttgart, 175 pp. 